\title{
Modelos Computacionais para Verificação de Identidades Polinomiais em Álgebras de Matrizes com entradas na Álgebra de Grassmann
}

\author{
R.M. SANTOS, S.M. ALVES e F.B.S. OLIVEIRA \\ Recebido em 05 de setembro de 2017 / Aceito em 23 de maio de 2018
}

\begin{abstract}
RESUMO. Nesse trabalho apresentamos uma abordagem computacional para tratar de álgebras que satisfazem identidades polinomiais. Mais precisamente, utilizamos o programa Maple para verificar e identificar identidades polinomiais das álgebras de matrizes com entradas na álgebra de Grassmann $E$, em especial a álgebra $M_{k, l}(E)$, a qual Di Vincenzo e La Scala apresentaram resultados interessantes quando $k=l=1$, usando a noção de identidades polinomiais fracas. Foram criados alguns procedimentos em Maple para adequar o produto das matrizes segundo as propriedades de $E$, sendo esta uma álgebra não comutativa. Além disso, implementamos algumas funções com menor tempo de processamento na solução de determinados problemas em comparação com funções análogas do Maple. Vale ressaltar que utilizamos o Maple na versão 18 em um computador com processador Intel $\AA$ Core ${ }^{\mathrm{TM}} \mathrm{i}$ e $8 \mathrm{~GB}$ de memória RAM. Finalizamos com o estudo da conjectura dada por Kemer acerca do grau mínimo do polinômio standard para a álgebra $M_{n}(E)$.
\end{abstract}

Palavras-chave: PI-álgebras; Maple; Computação Algébrica; Identidades Polinomiais Fracas.

\section{INTRODUÇÃO}

A teoria de álgebras com identidades polinomiais, ou simplesmente PI-álgebras, é uma teoria relativamente nova, tendo seu maior desenvolvimento ocorrido a partir de 1945, sobretudo com os trabalhos dos matemáticos N. Jacobson, J. Levitzki e I. Kaplansky (ver [14], [2] e [16]), que tratavam da estrutura de anéis (ou álgebras) com identidades polinomiais. Antes desse período, podemos destacar os trabalhos [5] e [26], considerados os primeiros a exibirem polinômios em variáveis não comutativas que se anulam quando avaliados por elementos de uma álgebra, ambos com motivação geométrica.

Do ponto de vista combinatório e computacional, como um dos primeiros trabalhos relevantes podemos citar [2], onde se prova que o polinômio standard de grau $2 n$ é a identidade de menor grau da álgebra $M_{n}(K)$, onde $K$ é um corpo de característica zero (denotamos por char $(K)=0$ ).

Autor correspondente: Rudhero Monteiro dos Santos - E-mail: rudhero@gmail.com

Departamento de Ciências Exatas e Tecnológicas, DCET, Universidade Estadual de Santa Cruz, Campus Soane Nazaré de Andrade, Rodovia Jorge Amado, Km 16, Salobrinho, 45662-900, Ilhéus, BA, Brasil. E-mail: rmsantos@gmail.com; smalves@uesc.br; fbsoliveira@uesc.br 
Tal resultado é conhecido como o teorema de Amitsur-Levitzki, um dos mais importantes para essa teoria tendo sido demonstrado de formas diferentes por vários pesquisadores.

Em [16] foi apresentada uma lista de problemas que motivaram significativamente a pesquisa da década. Um destes problemas diz respeito à existência de polinômios centrais para a álgebra das matrizes, $M_{n}(K)$ com $n>2$. Uma versão revisada é apresentada em [17]. A positivação do problema de Kaplansky foi dada independentemente em 1972 e 1973 por Formanek e Razmyslov em [9] e [23], e este fato foi de grande importância para a teoria dos anéis.

Alguns dos teoremas importantes que foram estabelecidos ou simplificados usando a noção de polinômios centrais podem ser consultados em [10], [15] e [24]. Combinando as ideias de Formanek e Razmyslov, vários polinômios centrais foram construídos em [13], [6] e [12].

A noção de identidade polinomial fraca foi introduzida em [22] visando resolver vários problemas, entre eles o de construir polinômios centrais para $M_{n}(K)$. Outro motivo foi estudar as identidades polinomiais ordinárias para $M_{n}(K)$.

Por exemplo, uma das observações de Razmyslov, em [22], resulta que as identidades polinomiais de $M_{2}(K)$, no caso em que $\operatorname{char}(K)=0$, seguem das identidades polinomiais de grau menor que 7 e que as identidades fracas de $M_{2}(K)$ são consequências da identidade

$$
\left[x_{1}^{2}, x_{2}\right]=0 .
$$

Um outro problema refere-se ao grau mínimo dos polinômios centrais para a álgebra de matrizes $M_{n}(K)$ quando a característica de $K$ é zero. Os polinômios centrais de Formanek, encontrados em [9], são de grau $n^{2}$. Os polinômios obtidos por Razmyslov são de grau $3 n^{2}-1$. Usando a noção de identidade fraca, Halpin reduziu os polinômios de Razmyslov para polinômios de grau $n^{2}$. Em posse dessas informações, Formanek conjecturou que o grau mínimo dos polinômios centrais para $M_{n}(K)$ sobre um corpo $K$ de característica zero é dada por:

$$
\operatorname{mindeg}\left(M_{n}(K)\right)=\frac{1}{2}\left(n^{2}+3 n-2\right) .
$$

Um fato importante é que a conjectura de Formanek está diretamente relacionada com vários problemas em aberto na teoria de álgebras com identidades polinomiais.

Para $n=4$, V. Drensky e G. Cattaneo, em [7], encontraram um polinômio central de grau $13=$ $\frac{1}{2}\left(4^{2}+3 \cdot 4-2\right)$. Mas não sabemos se existem polinômios centrais de grau 12 para $M_{4}(K)$. Eles usaram identidades fracas de grau 8 e 9, e os métodos de Razmyslov para obter o resultado, o qual, em [6], foi generalizado para construir polinômios de grau $(n-1)^{2}+4$ para $M_{n}(K)$ com $n>2$.

Consequentemente, surge o problema acerca do grau mínimo para uma identidade polinomial fraca da álgebra de matrizes $M_{n}(K)$ quando $n>2$. Este foi positivado para $n=2 \mathrm{em} \mathrm{[23],} \mathrm{onde} \mathrm{é}$ mostrado que todas as identidades polinomiais fracas de $M_{2}(K)$ são consequências da identidade fraca da Equação 1.1.

Para $n=3$, V. Drensky e T. G. Raskova, em [8], descreveram todas as identidades polinomiais fracas de grau 6, as quais deram lugar a polinômios centrais de grau 8 e 9 . 
Nesse trabalho, buscamos resultados parecidos para as álgebras $M_{n}(E)$ e $M_{k, l}(E)$ (ver a Equação 2.1), sendo $E$ a álgebra de Grassmann sobre um corpo de característica positiva diferente de 2 .

\section{NOÇÕES PRELIMINARES}

Nessa seção apresentamos algumas definições e resultados inerentes ao estudo de Álgebras com Identidades Polinomiais, ou simplesmente PI-álgebras. Apresentamos inicialmente o conceito de álgebra, nosso principal objeto, e o de identidades polinomiais, seguidos de alguns exemplos.

\section{1 Álgebras}

No texto que segue, $K$ denotará um corpo e, a menos que se diga o contrário, todas as álgebras e espaços vetoriais serão definidos sobre o corpo $K$.

Definition 2.1. Seja A um espaço vetorial sobre K munido de uma operação binária $*: A \times A \longrightarrow$ A. Dizemos que A é uma K-álgebra (álgebra sobre K ou simplesmente álgebra) se para qualquer $\alpha \in K$ e quaisquer $a, b, c \in A$, valerem as seguintes propriedades:

1. $(a+b) * c=a * c+b * c ;$

2. $a *(b+c)=a * b+a * c$;

3. $\alpha(a * b)=(\alpha a) * b=a *(\alpha b)$.

Em outras palavras, para que $A$ seja uma álgebra, basta que tenha estrutura de espaço vetorial e seja munida de uma operação bilinear, a qual chamamos de multiplicação. Na definição anterior, usamos o símbolo * para a multiplicação de dois elementos de $A$ a fim de distingui-la da multiplicação por escalar, mas a partir de agora, para simplificar a notação, denotaremos ambas as operações do mesmo modo, isto é, $a * b$ será escrita como $a b$.

Definition 2.2. Uma álgebra A é dita ser

1. comutativa, se $a b=$ ba para quaisquer $a, b \in A$;

2. associativa, se $(a b) c=a(b c)$ para quaisquer $a, b, c \in A$;

3. unitária, se existir $1_{A} \in A$ chamado de elemento identidade de A tal que $1_{A} a=a 1_{A}=a$, para qualquer $a \in A$ (escreveremos 1 em vez de $1_{A}$ ).

Analogamente às demais estruturas algébricas, podemos definir homomorfismos de álgebras, bem como mergulhos, isomorfismos, automorfismos, dentre outras.

Destacamos os seguintes exemplos de álgebras de nosso interesse:

1. Seja $V$ um $K$-espaço vetorial com base enumerável $\left\{e_{i} \mid i \in \mathbb{N}\right\}$. A álgebra de Grassmann (ou exterior) $E=E(V)$ é a álgebra tendo como base $\beta=\left\{1, e_{i_{1}} e_{i_{2}} \cdots e_{i_{k}} \mid i_{1}<i_{2}<\ldots<\right.$ 
$\left.i_{k}, k \geq 1\right\}$ satisfazendo as relações $e_{i} e_{j}=-e_{j} e_{i}$ e $e_{i}^{2}=0$, para todos $i, j \in \mathbb{N}$. Se $\operatorname{char}(K) \neq$ 2 essa última condição segue da primeira. Além disso, destacamos em $E$ os seguintes subespaços vetoriais:

(a) $E_{0}$, gerado pelo conjunto

$$
\beta_{0}=\left\{1, e_{i_{1}} e_{i_{2}} \cdots e_{i_{m}} \mid i_{1}<i_{2}<\ldots<i_{m}, m=2,4,6, \ldots\right\}
$$

(b) $E_{1}$, gerado pelo conjunto

$$
\beta_{1}=\left\{e_{i_{1}} e_{i_{2}} \cdots e_{i_{k}} \mid i_{1}<i_{2}<\ldots<i_{k}, k=1,3,5, \ldots\right\}
$$

2. O $K$-espaço vetorial das matrizes $n \times n$ com entradas na álgebra de Grassmann $E$, denotado por $M_{n}(E)$, munido com a multiplicação usual de matrizes tem estrutura de álgebra.

Claramente, $E=E_{0} \oplus E_{1}$ como espaço vetorial. Uma vez que $e_{i} e_{j}=-e_{j} e_{i}$ temos $\left(e_{i_{1}} e_{i_{2}} \cdots e_{i_{m}}\right)\left(e_{j_{1}} e_{j_{2}} \cdots e_{j_{k}}\right)=(-1)^{m k}\left(e_{j_{1}} e_{j_{2}} \cdots e_{j_{k}}\right)\left(e_{i_{1}} e_{i_{2}} \cdots e_{i_{m}}\right)$, para quaisquer $m, k \in \mathbb{N}$ e, assim, podemos concluir que $a x=x a$ para quaisquer $a \in E_{0}$ e $x \in E$, e $b c=-c b$ para quaisquer $b, c \in E_{1}$.

Note que se $\operatorname{char}(K)=2$, então $E$ é uma álgebra comutativa. Além disso, considerando $E^{\prime}$ a álgebra com base $\beta-\{1\}$, temos que $E^{\prime}$ não tem elemento identidade e é chamada de álgebra Grassmann sem elemento identidade.

Definition 2.3. Sejam A uma álgebra e B um $K$-subespaço vetorial de A. Dizemos que $B$ é uma K-subálgebra de A, se tiver estrutura de álgebra, isto é, se $B$ for fechado com respeito à multiplicação definida em $A$. $O$ subespaço $B$ é denominado um ideal à esquerda de $A$ se $A B \subset B$. De modo similar, definimos ideal à direita de A. Um ideal bilateral (ideal à direita e à esquerda simultaneamente) será simplesmente denominado de ideal.

Destacamos os seguintes exemplos de subálgebras de nosso interesse:

1. Seja $A$ uma álgebra. $\mathrm{O}$ subconjunto

$$
Z(A)=\{a \in A \mid a b=b a, \text { para qualquer } b \in A\}
$$

é uma subálgebra de $A$, o qual denominamos de centro de $A$ e seus elementos são ditos centrais. Note que se $A=E$ temos que $Z(E)=E_{0}$, isto é, $E_{0}$ é o centro da álgebra de Grassmann.

2. Se $k, l \in \mathbb{N}$ são tais que $k+l=n$, é fácil verificar através de multiplicação de matrizes, que o $K$-subespaço de $M_{a+b}(E)$ dado por

$$
M_{k, l}(E)=\left\{\left(\begin{array}{cc}
A & B \\
C & D
\end{array}\right) \mid \begin{array}{l}
A \in M_{k}\left(E_{0}\right), B \in M_{k \times l}\left(E_{1}\right), \\
C \in M_{l \times k}\left(E_{1}\right), D \in M_{l}\left(E_{0}\right)
\end{array}\right\}
$$

é uma subálgebra de $M_{k+l}(E)$. 
Vale ressaltar a importância das propriedades das álgebras apresentadas nos exemplos supracitados para a implementação dos procedimentos no Maple na obtenção de identidades polinomiais, sobre as quais discorremos a seguir.

\section{2 Álgebras com Identidades Polinomiais}

Seja $X=\left\{x_{1}, x_{2}, \ldots\right\}$ um conjunto não vazio e enumerável de variáveis não comutativas. Uma palavra em $X$ é uma sequência $x_{1} x_{2} \ldots x_{n}$ onde $n \in \mathbb{N}$ e $x_{i_{j}} \in X$ (para $n=0$ temos a palavra vazia e vamos denotá-la por 1). Considerando $S(X)$ o conjunto de todas as palavras em $X$ e definindo que duas palavras $x_{i_{1}} x_{i_{2}} \ldots x_{i_{n}}$ e $x_{j_{1}} x_{j_{2}} \ldots x_{j_{m}}$ são iguais se $n=m$ e $i_{1}=j_{1}, i_{2}=j_{2}, \ldots, i_{n}=j_{n}$, denotamos por $K\langle X\rangle$ o espaço vetorial que tem como base o conjunto $S(X)$. Os elementos de $K\langle X\rangle$ são, portanto, somas (formais) de termos que são produtos (formais) de um escalar por uma palavra em $X$.

Definindo em $S(X)$ a seguinte multiplicação

$$
\left(x_{i_{1}} \ldots x_{i_{n}}\right)\left(x_{j_{1}} \ldots x_{j_{m}}\right)=x_{i_{1}} \ldots x_{i_{n}} x_{j_{1}} \ldots x_{j_{m}}, \text { para quaisquer } x_{i_{k}}, x_{j_{l}} \in X
$$

é fácil mostrar que $K\langle X\rangle$, com essa multiplicação, é uma álgebra associativa livre com elemento identidade (a palavra vazia) (ver definição de álgebra livre em [11]).

Os produtos de um escalar por uma palavra são chamados de monômios e os elementos de $K\langle X\rangle$ são chamados de polinômios. Notemos que a álgebra $K\langle X\rangle$ é, em outras palavras, a álgebra dos polinômios não-comutativos.

Definition 2.4. Um monômio $m$ tem grau $k$ em $x_{i}$ se a variável $x_{i}$ ocorre em $m$ exatamente $k$ vezes. Um polinômio é homogêneo de grau $k$ em $x_{i}$ se todos os seus monômios têm grau $k$ em $x_{i}$. Denotamos este fato por $\operatorname{deg}_{x_{i}} f=k$. Um polinômio linear em $x_{i}$ é um polinômio de grau 1 em $x_{i}$.

Definition 2.5. Um polinômio é multihomogêneo se para cada variável $x_{i}$ todos os seus monômios têm o mesmo grau em $x_{i}$. Um polinômio é multilinear se é linear em cada variável. $O$ grau de um polinômio é o maior grau entre todos os seus monômios.

Sejam $f$ um polinômio de $K\langle X\rangle$ de grau $n$ e $x_{k}$ uma variável de $f$. Podemos escrever $f$ como uma soma $f=\sum_{i=0}^{n} f_{i}$, onde cada polinômio $f_{i}$ é homogêneo de grau $i$ na variável $x_{k}$. Cada polinômio $f_{i}$ é chamado de componente homogênea de grau $i$ em $x_{k}$ do polinômio $f$.

Os polinômios multilineares e multihomogêneos desempenham um papel importante na busca de bases para as identidades polinomiais sobre determinados tipos de corpos. Quando a álgebra é unitária podemos restringir a nossa busca de identidades polinomiais a um determinado tipo de polinômio que vamos definir nos próximos exemplos.

Destacamos alguns tipos especiais de polinômios: 
1. O comutador de comprimento $n$ é definido indutivamente a partir de $\left[x_{1}, x_{2}\right]=x_{1} x_{2}-x_{2} x_{1} \mathrm{e}$ tomando $\left[x_{1}, x_{2}, \ldots, x_{n}\right]=\left[\left[x_{1}, x_{2}, \ldots, x_{n-1}\right], x_{n}\right]$ para $n>2$. Um polinômio $f \in K\langle X\rangle$ é chamado polinômio próprio, se ele for uma combinação linear de produtos de comutadores, isto é,

$$
f\left(x_{1}, x_{2}, \ldots, x_{n}\right)=\alpha_{i, \ldots, j}\left[x_{i_{1}}, \ldots, x_{i_{p}}\right] \ldots\left[x_{j_{1}}, \ldots, x_{j_{q}}\right] \operatorname{com} \alpha_{i, \ldots, j} \in K
$$

(Assumimos que 1 é o produto de um conjunto vazio de comutadores).

2. O polinômio

$$
s t_{n}\left(x_{1}, \ldots, x_{n}\right)=\sum_{\sigma \in S_{n}}(-1)^{\sigma} x_{\sigma(1)} \ldots x_{\sigma(n)}
$$

é chamado polinômio standard de grau $n$. Aqui $(-1)^{\sigma}$ é o sinal da permutação $\sigma$ do grupo simétrico $S_{n}$.

Agora, podemos definir o conceito de identidade polinomial.

Definition 2.6. Um polinômio $f\left(x_{1}, \ldots, x_{n}\right) \in K\langle X\rangle$ é denominado uma identidade polinomial da álgebra $A$, se $f\left(a_{1}, \ldots, a_{n}\right)=0$, para quaisquer $a_{1}, \ldots, a_{n} \in A$. Uma álgebra com identidade polinomial (PI-álgebra) é uma álgebra que satisfaz uma identidade polinomial não trivial (polinômio não nulo).

Observemos que $f=f\left(x_{1}, \ldots, x_{n}\right)$ é uma identidade de $A$ se, e somente se, $f$ pertence aos núcleos de todos os homomorfismos de $K\langle X\rangle$ em $A$. Denotamos por $T(A)$ o conjunto de todas as identidades polinomiais de $A$, e assim, dizemos que $A$ é uma PI-álgebra se $T(A) \neq\{0\}$.

A seguir, apresentamos alguns exemplos de PI-álgebras, as quais verificaremos com procedimentos no Maple na seção seguinte.

1. Toda álgebra comutativa $A$ é uma PI-álgebra, pois o polinômio comutador $f\left(x_{1}, x_{2}\right)=$ $\left[x_{1}, x_{2}\right]=x_{1} x_{2}-x_{2} x_{1}$ é uma identidade polinomial para $A$.

2. A álgebra de Grassmann $E$ é uma PI-álgebra, pois um cálculo direto usando os elementos da base de $E$ mostra que o polinômio comutador $f\left(x_{1}, x_{2}, x_{3}\right)=\left[\left[x_{1}, x_{2}\right], x_{3}\right]$ é uma identidade polinomial para $E$.

3. A álgebra $M_{2}(K)$ satisfaz a identidade $f\left(x_{1}, x_{2}, x_{3}\right)=\left[\left[x_{1}, x_{2}\right]^{2}, x_{3}\right]$, conhecida como a identidade de Hall.

4. (Teorema de Amitsur-Levitzki) A álgebra $M_{n}(K)$ satisfaz o polinômio standard de grau $2 n$

$$
s t_{2 n}\left(x_{1}, \ldots x_{2 n}\right)=\sum_{\sigma \in S_{2 n}}(-1)^{\sigma} x_{\sigma(1)}, \ldots x_{\sigma(2 n)}
$$

onde $S_{2 n}$ é o grupo das permutações de $\{1,2, \ldots, 2 n\}$ e $(-1)^{\sigma}$ é o sinal da permutação $\sigma$. 
Note que, devido às propriedades do grupo $S_{n}$, temos que o polinômio standard é identicamente nulo sempre que duas de suas variáveis são iguais, o que nos permite enunciar o seguinte teorema, cuja demonstração vamos omitir (ver [11]).

Theorem 1. Seja A uma álgebra de dimensão n sobre um corpo K. Então, A satisfaz o polinômio standard de grau $n+1$.

Um exemplo de uma álgebra que não satisfaz identidades polinomiais não nulas é a álgebra $K\langle X\rangle$, o que pode ser compreendido por um argumento simples. Suponhamos, por absurdo, que $f\left(x_{1}, \ldots, x_{n}\right)$ seja uma identidade polinomial não nula de $K\langle X\rangle$. Assim, $f\left(f_{1}\left(x_{1}\right), \ldots, f_{n}\left(x_{n}\right)\right)=0$, onde $f_{i}\left(x_{i}\right)=x_{i}$ para $i=1, \ldots, n$, o que é um absurdo, pois $f\left(x_{1}, \ldots, x_{n}\right) \neq 0$. Outro exemplo, facilmente verificado, é a álgebra dos operadores lineares de posto finito de um espaço vetorial $V$, de dimensão infinita.

O exemplo da Equação 2.5 foi muito importante para auxiliar na implementação computacional do polinômio standard de grau $n$ para a álgebra $M_{k, l}(E)$, o qual é objeto central do nosso trabalho. Alguns exemplos dos procedimentos criados, bem como aplicação dos mesmos é mostrado na seção a seguir.

\section{PROCEDIMENTOS EM MAPLE PARA VERIFICAR IDENTIDADES POLINOMIAIS}

Na seção anterior, apresentamos alguns exemplos de álgebras satisfazendo identidades polinomiais. Nesta, discorremos sobre o Maple e apresentamos alguns procedimentos computacionais para verificar a validade de tais identidades, bem como encontrar identidades novas.

\subsection{Sobre o Maple}

Para mais detalhes sobre o Maple indicamos [21], o guia de ajuda disponibilizado no próprio programa ou online ${ }^{1}$ e, também, o livro [3] que apresenta uma boa introdução à computação algébrica com Maple tratando de diversos temas da matemática. Os procedimentos apresentados nessa seção foram construídos com a versão 18 do programa, instalado em um computador com processador Intel(B) Core i7 e 8GB de memória RAM.

Nesse trabalho, utilizamos diversos procedimentos simbólicos de álgebra abstrata, o que justifica o uso do Maple. A parte do programa que não compõe o núcleo, consiste de duas: a biblioteca principal e um conjunto de vários pacotes (packages) separados. Assim como os comandos que fazem parte do núcleo, os comandos da biblioteca principal são carregados automaticamente na hora da inicialização e estão, de imediato, prontos para serem usados.

Além das diversas funções implementadas no Maple para se trabalhar com matemática simbólica, evidenciamos o fato de o mesmo possuir um operador não comutativo (dot . ), próprio para tratar de matrizes, vetores e outros objetos em que não vale a propriedade comutativa, o que

${ }^{1}$ Acessar o site http://www.maplesoft.com/support/help 
o torna ainda mais interessante para o nosso trabalho, visto que estudamos álgebras com tais propriedades. Vale ressaltar que esse operador é associativo e possui o 1 como elemento neutro, porém não vale a distributividade em relação à soma.

Para acrescentar a propriedade distributiva em relação à soma do operador dot recorremos à programação no Maple.

Um procedimento (ou programa) no Maple é essencialmente um conjunto de comandos (do próprio Maple ou estruturas usuais de programação if, else, for, while, entre outros) organizados de forma sequenciada e lógica, sendo executados manualmente ou de forma automática na ordem em que aparecem da esquerda para a direita e de cima para baixo.

Procedimento: distributiva à direita com o operador . (dot)

Entrada: Duas expressões $x_{1}+x_{2}+\ldots+x_{n}$ e $y_{1}+y_{2}+\ldots+y_{n}$.

Saída: Uma expressão $x_{1} \cdot y_{1}+\ldots+x_{i} \cdot y_{j}+\ldots+x_{n} \cdot y_{n}$.

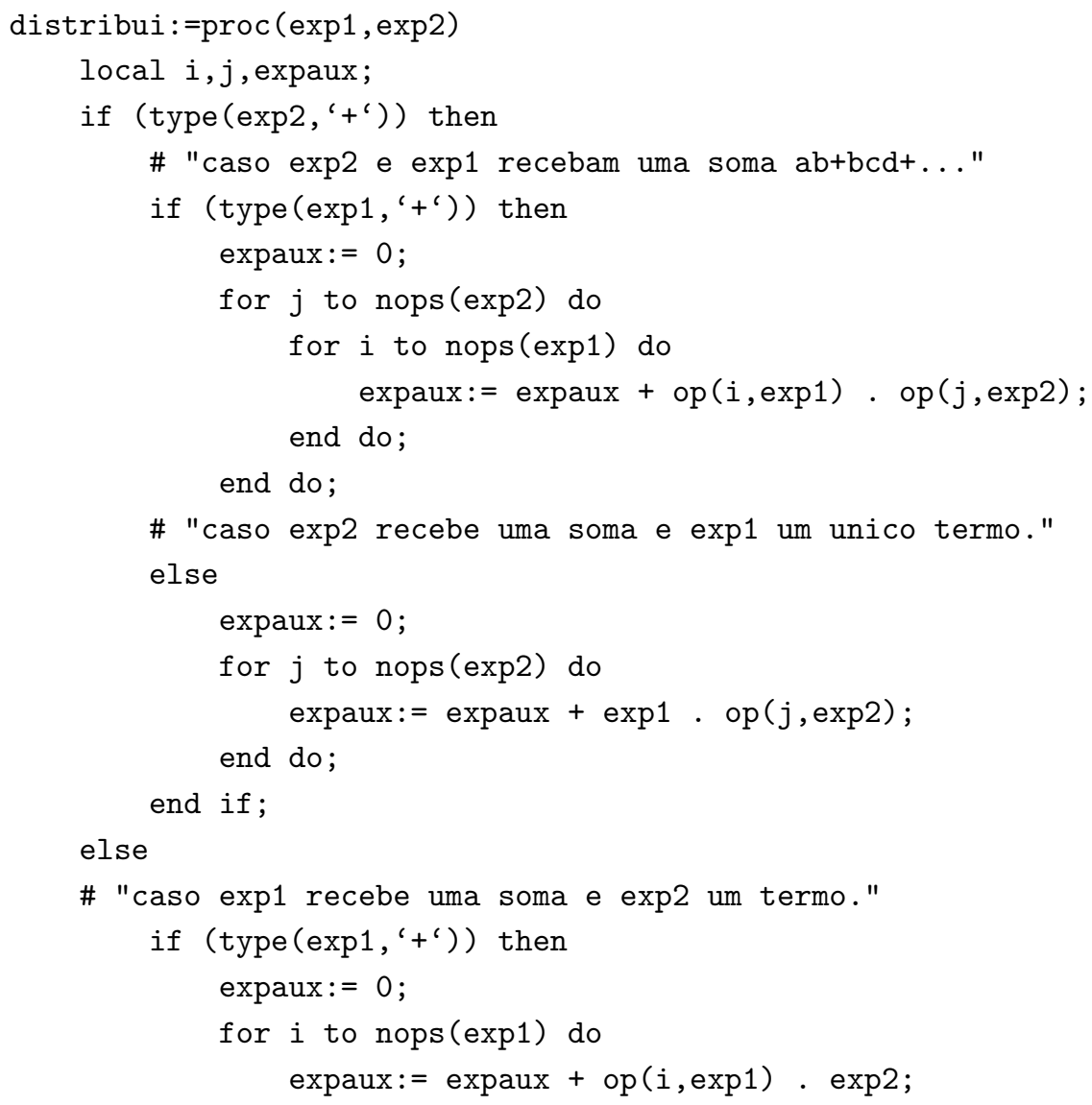




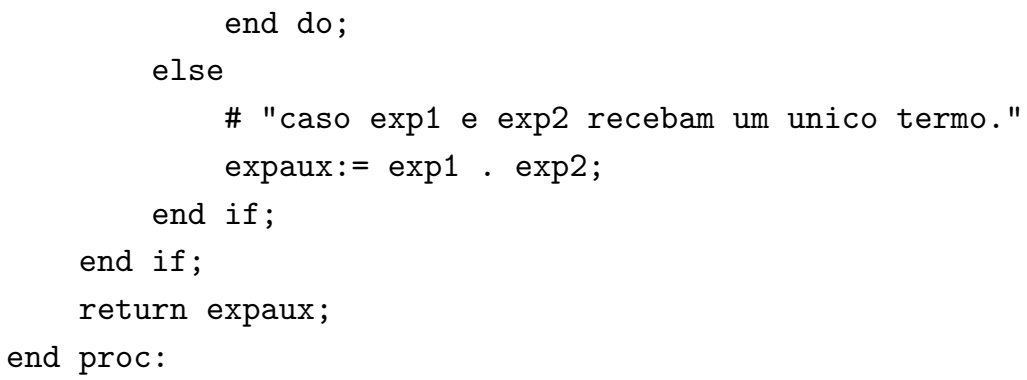

Nesse procedimento, as variáveis $i, j$ são utilizadas como controladoras de estruturas de repetição e expaux armazena a expressão de saída. Cada caso está devidamente comentado após o símbolo \#, onde tudo que for escrito na mesma linha é desconsiderado pelo compilador do Maple. Além disso, caso se queira um bloco de comentários pode-se usar a estrutura (*comentários *).

Note que não precisamos declarar o tipo das variáveis dentro de um procedimento no Maple. A função type identifica automaticamente diversos tipos de variáveis que podem ser numéricas, algébricas, simbólicas ou até mesmo outros procedimentos. Porém, deve-se ter cuidado quanto à omissão do tipo da variável, visto que os procedimentos podem gerar resultados errados (ou não esperados) a partir de alguns dados de entrada.

Como dito no início desta seção, o Maple possui vários pacotes separados que podem ser carregados na folha de trabalho com o comando with(nome do pacote) para que se tornem disponíveis algumas funções pré-implementadas. Alguns dos pacotes de nosso interesse foram $\mathrm{Li}$ nearAlgebra, group, combinat e Physics, que permitiram trabalhar com manipulação de matrizes, permutações, análise combinatória, entre outros. O pacote Physics merece uma atenção especial, visto que o mesmo permite trabalhar de forma simples com álgebra não comutativa. Por isso, tratamos algumas de suas principais funções que usamos no presente estudo.

Nesse pacote, existe uma boa quantidade de funções disponíveis, dentre elas destaca-se a função Setup que é usado para definir, limpar e consultar o ambiente computacional usado pelos comandos do pacote Physics.

Com a função Setup, podemos definir as variáveis como não comutativas ou anticomutativas com auxílio dos parâmetros noncommutativeprefix e anticommutativeprefix, respectivamente. Além disso, para utilizar a notação habitual de matemática, usamos o parâmetro mathematicalnotation atribuindo valores lógicos (true ou false) dentro da função Setup. Além destas, vale destacar também as funções Commutator e AntiCommutator, as quais usamos em diversos procedimentos.

\subsection{A Identidade $\left[\left[x_{1}, x_{2}\right], x_{3}\right]$ da Álgebra de Grassmann}

Na seção 3.1, vimos que existe uma função do Maple no pacote Physics, denominada Commutator, que calcula o comutador entre duas variáveis. Em notação matemática do Maple, o comuta- 
dor de duas variáveis $x, y$ é dado por $[x, y]_{-}=x y-y x$, visto que é usada uma notação semelhante para o anticomutador (função AntiCommutator) dado por $[x, y]_{+}=x y+y x$. Vale lembrar que são usados apenas dois parâmetros em cada função.

Logo, assumindo que $x_{1}, x_{2}, x_{3} \in E_{1}$ (Se alguma variável for de $E_{0}$ a identidade é óbvia, pois o comutador entre duas delas será nulo), podemos verificar a identidade da seguinte forma:

with(Physics):

Setup $($ mathematicalnotation $=$ true, anticommutativeprefix $=x)$ :

$f:=\operatorname{Commutator}(x[1], x[2])$

$$
\left[x_{1}, x_{2}\right]_{-}
$$

$f:=$ Commutator $(f, x[3])$

Porém, um teste de eficiência (ver Tabela 1) da função Commutator mostra que a mesma não é uma boa opção para o simples cálculo de comutadores de comprimento maior que 6 quando as variáveis são não comutativas. Em virtude disso, implementamos funções independentes do pacote Physics, para resolver esse e outros problemas.

Vale lembrar que o comutador de comprimento $n=2$ é dado por $\left[x_{1}, x_{2}\right]=x_{1} x_{2}-x_{2} x_{1}$, ou seja, para $n>2$ basta tomar a diferença entre a distributiva do elemento $x_{i}$ à esquerda e à direita da expressão anterior, para cada $i=3,4, \ldots, n$. Logo, podemos utilizar o procedimento apresentado na seção 3.1 .

Procedimento: comutador (colchete de Lie).

Entrada: Uma lista $L:=\left[x_{1}, x_{2}, \ldots, x_{n}\right] \operatorname{com} n \geq 2$.

Saída: Uma expressão dada pelo colchete de Lie.

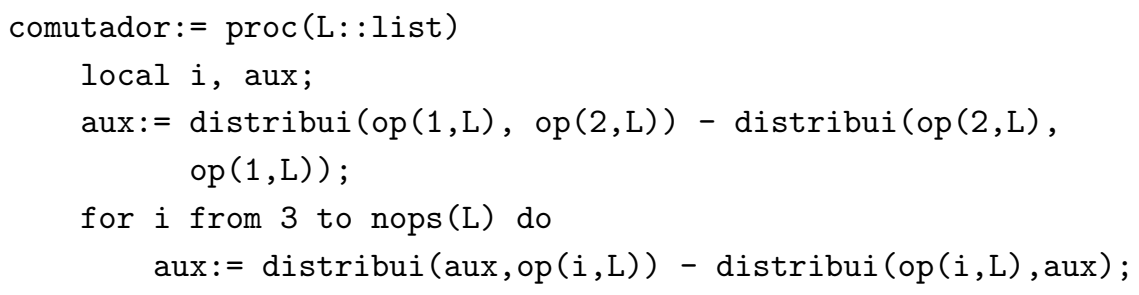


end do;

return aux;

end proc:

Neste procedimento, declaramos o tipo de entrada de dados (list) usando o símbolo :: e usamos a variável $i$ como controlador de loop, enquanto aux armazena a expressão de saída. Note que utilizamos um procedimento dentro do outro, o que permitiu uma redução de linhas de comandos no mesmo.

Usando tais procedimentos (que devem ser executados na mesma folha de trabalho) podemos, por exemplo, reescrever a identidade $f\left(x_{1}, x_{2}, x_{3}\right)=\left[\left[x_{1}, x_{2}\right], x_{3}\right]$ de $E$ entrando com os seguintes comandos no Maple

$f:=[x[1], x[2], x[3]]:$

comutador(f);

$$
x_{1} \cdot x_{2} \cdot x_{3}-x_{2} \cdot x_{1} \cdot x_{3}-x_{3} \cdot x_{1} \cdot x_{2}+x_{3} \cdot x_{2} \cdot x_{1}
$$

Note que obtemos um resultado semelhante ao anterior, exceto por questões de notação (o operador . fica visível), sem usar o Setup. Calculamos o tempo real gasto para obter o comutador de comprimento $n=2, \ldots, 10$ usando as funções comutador e Commutator, separadamente. Para isso, utilizou-se a função time [real]() que retorna o tempo real decorrido desde a inicialização do núcleo do Maple, em segundos, truncado em apenas 3 casas decimais. Os resultados aparecem na Tabela 1.

Note que, a partir de $n=6$ a função Commutator passa a ser muito lenta. Porém, apesar de calcular de forma mais rápida, a função comutador não leva em consideração nenhuma propriedade da variável (lembrando que o operador . não comuta as variáveis). Assim, caso queiramos que as variáveis anticomutem, isto é, $x_{1} x_{2}=-x_{2} x_{1}$, precisamos criar um novo procedimento, o qual denominamos de ordenaEl e omitimos seu código nesse texto.

Como exemplo de aplicação da função, utilizamos o procedimento supracitado em cada termo da expressão obtida pelo comutador $(f)$ (Nesse caso, os dois procedimentos devem ser executados na mesma folha de trabalho) da seguinte forma

$$
f:=[x[1], x[2], x[3]]:
$$


Tabela 1: Tempo (em segundos) para calcular o comutador de comprimento $n$

\begin{tabular}{ccc}
\hline $\mathbf{n}$ & Usando comutador & Usando Commutator \\
\hline 2 & $1 \mathrm{E}-3$ & $1 \mathrm{E}-3$ \\
3 & $1 \mathrm{E}-3$ & $1 \mathrm{E}-3$ \\
4 & $2 \mathrm{E}-3$ & $6 \mathrm{E}-3$ \\
5 & $7 \mathrm{E}-3$ & $1 \mathrm{E}-2$ \\
6 & $1.4 \mathrm{E}-2$ & $3 \mathrm{E}-2$ \\
7 & $4 \mathrm{E}-2$ & $1.2941 \mathrm{E} 1$ \\
8 & $6.7 \mathrm{E}-2$ & $4.737 \mathrm{E} 1$ \\
9 & $1.32 \mathrm{E}-1$ & $2.55582 \mathrm{E} 2$ \\
10 & $2.75 \mathrm{E}-1$ & $4.31738 \mathrm{E} 2$ \\
\hline
\end{tabular}

$f:=$ comutador $(f)$ :

$\operatorname{aux}:=0$ :

for i to nops $(f)$ do aux: $=$ aux $+\operatorname{ordenaE1}(\operatorname{op}(i, f))$; end do:

aux;

Observe que isso é suficiente para mostrar que $f\left(x_{1}, x_{2}, x_{3}\right)=\left[\left[x_{1}, x_{2}\right], x_{3}\right]=0$, para qualquer $x_{i} \in$ $E$. Portanto $E$ é uma PI-álgebra. Note que, até então, nada foi dito sobre o uso da propriedade $x_{i}^{2}=$ 0 , para qualquer $x_{i} \in E_{1}$. Para satisfazer tal propriedade foi necessário mais um procedimento, o qual denominamos de ordenaE1E1, o que se faz necessário quando tomamos polinômios que não são multilineares como, por exemplo, o polinômio da Equação 1.1.

\subsection{A Identidade de Hall $\left[\left[x_{1}, x_{2}\right]^{2}, x_{3}\right]$}

Agora, vamos verificar que o polinômio $\left[\left[x_{1}, x_{2}\right]^{2}, x_{3}\right]$ é uma identidade polinomial para as matrizes quadradas de ordem 2 sobre $K$.

Considere $f\left(x_{1}, x_{2}, x_{3}\right)=\left[\left[x_{1}, x_{2}\right]^{2}, x_{3}\right]$. Para calcular $\left[x_{1}, x_{2}\right]^{2}$ basta combinarmos os procedimentos comutador e distribui da seguinte forma

for i to 3 do $x[i]:=\operatorname{Matrix}([[a[i], b[i]],[c[i], d[i]]])$; end do; 


$$
\left[\begin{array}{ll}
a_{1} & b_{1} \\
c_{1} & d_{1}
\end{array}\right],\left[\begin{array}{ll}
a_{2} & b_{2} \\
c_{2} & d_{2}
\end{array}\right],\left[\begin{array}{ll}
a_{3} & b_{3} \\
c_{3} & d_{3}
\end{array}\right]
$$

$f:=\operatorname{comutador}([x[1], x[2]]):$

$f:=\operatorname{distribui(f,f):~\# ~ou~apenas~} f:=f . f$ :

$f:=$ comutador $([f, x[3]]):$

$\operatorname{simplify}(\%)$;

$$
\left[\begin{array}{ll}
0 & 0 \\
0 & 0
\end{array}\right]
$$

Como as entradas das matrizes são elementos de um corpo (logo comutam entre si) o Maple nos dá um resultado direto apenas com os procedimentos construídos até aqui. Nesse caso, o comando simplify faz as simplificações necessárias de acordo com as propriedades do corpo (associatividade, distributividade, comutatividade, etc.) e mostra que $f$ é, de fato, uma identidade polinomial de $M_{2}(K)$. O tempo médio foi de 1.48 segundos. De forma análoga, usando a função Commutator obtemos o resultado após 1.65 segundos. Mas, esta função seria interessante caso as entradas das matrizes fossem não comutativas, como veremos na próxima seção.

\section{O POLINÔMIO STANDARD PARA $M_{K, L}(E)$}

Em [25], Di Vincenzo e La Scala apresentaram resultados interessantes para a álgebra $M_{k, l}(E)$, quando $k=l=1$, onde os autores definem e usam a noção de identidades polinomiais fracas para encontrar os polinômios $c=\left[x_{1}, x_{2}, x_{3}\right]$ e $p=\left[x_{2}, x_{1}\right]\left[x_{3}, x_{1}\right]\left[x_{4}, x_{1}\right]$. De forma geral, Popov, em [20], encontrou os polinômios $\left[\left[x_{1}, x_{2}\right]^{2}, x_{2}\right]$ e $\left[x_{1}, x_{2},\left[x_{3}, x_{4}\right], x_{5}\right]$ que formam uma base para $T\left(M_{1,1}(E)\right)$. Nessa perspectiva, também citamos [19]. As identidades supracitadas foram verificadas e atestadas com os nossos procedimentos, porém não as descrevemos no presente texto. Nesta seção, nos limitamos aos resultados obtidos referente ao grau mínimo do polinômio standard para $M_{k, l}(E)$, em especial quando $k=l=1$.

Em [1], é dada uma condição necessária para que o polinômio standard de grau $m$ seja uma identidade polinomial de $M_{n}(E)$.

No artigo supracitado são demonstrados os seguintes resultados:

Lemma 1. Seja char $(K)=p$. O polinômio standard $S t_{m}$ é uma identidade polinomial de $E$ se, e somente se, $m \geq p+1$. 
Lemma 2. Se uma álgebra A satisfaz o polinômio standard $S t_{2 m}$, então $M_{n}(A)$ satisfaz o polinômio standard $S t_{2 m n^{2}-n^{2}+1}$.

Theorem 3. Seja $S t_{m}$ uma identidade polinomial para a álgebra $M_{n}(E)$. Então $m \geq 2 n+p-1$.

Pelo Lema 1, temos que $E$ satisfaz a identidade standard $S t_{p+1}$, o que implica que pelo Lema 2, $M_{n}(E)$ satisfaz $S t_{(p+1) n^{2}-n^{2}+1}=S t_{p n^{2}+1}$.

Dessa forma, questiona-se a existência de alguma identidade polinomial standard para $M_{n}(E)$ de grau $m$ tal que $m \in\left[2 n+p-1, p n^{2}+1\right]$. Em caso afirmativo, a busca pelo grau mínimo passa a ser interessante.

Em [18] encontramos a seguinte conjectura:

Seja $d_{n}$ o menor número $m$ tal que a álgebra $M_{n}(E)$ satisfaz a identidade standard de grau m. Então, $d_{n}=(2 n-1) p+1$.

Uma vez que $M_{k, l}(E) \subseteq M_{n}(E)$, então toda identidade polinomial de $M_{n}(E)$ é também uma identidade de $M_{k, l}(E)$, isto é, vale a inclusão $T\left(M_{n}(E)\right) \subseteq T\left(M_{k, l}(E)\right)$. Por conta disso, criamos alguns procedimentos no Maple para verificar a conjectura supracitada, considerando inicialmente a álgebra definida na Equação 2.1. É claro que o polinômio standard de grau $m<4$ não é uma identidade para $M_{k, l}(E)$, mas também as calculamos a fim de comparar os resultados obtidos com uso dos procedimentos construídos e das funções já implementadas do pacote Physics. Para este, bastou usar o procedimento que denominamos de Stn (calcula o polinômio standard de grau $n$ ) com uso da função setup (ver seção 3.1), de forma que se defina as entradas das matrizes $b$ e $c$ como sendo anticomutativas. Assim, usamos os comandos abaixo para obter os resultados da tabela a seguir:

$n:=[\operatorname{seq}(i, i=2 . .9)]:$

for $i$ to nops $(n)$ do

for $k$ to $n[i] d o$

$M[k]:=\operatorname{Matrix}(n[i], n[i],[[a[k], b[k]],[c[k], d[k]]]):$

end do:

$L:=[\operatorname{seq}(M[k], k=1 . . n[i])]:$

tempo[i]:= time $[$ real $]()$;

$A[i]:=\operatorname{Stn}(L)$;

tempo[i]:= time $[\mathrm{real}]()$-tempo $[i]$;

end do: 
Desse modo, conseguimos os resultados da seguinte tabela

Tabela 2: Tempo (em segundos) para calcular o polinômio standard de grau $n$

\begin{tabular}{ccc}
\hline $\mathbf{n}$ & Procedimentos próprios & Pacote Physics \\
\hline 2 & 0.128 & 0.228 \\
3 & 0.192 & 0.406 \\
4 & 2.594 & 4.067 \\
5 & 34.910 & 34.696 \\
6 & 479.861 & 640.708 \\
7 & 1023.358 & 1901.246 \\
8 & 21974.312 & - \\
9 & 529054.659 & - \\
\hline
\end{tabular}

Note que a partir de $n=6$, temos uma diferença considerável entre os procedimentos que construímos com os resultados obtidos com o pacote Physics. Uma vez que o processo para encontrar o polinômio standard torna-se muito lento devido às propriedades do grupo $S_{n}$, para $6<n<10$, não utilizamos as funções do pacote supracitado, o que justifica a ausência dos valores da Tabela 2 .

Veja que acumulamos cada polinômio standard de grau $i$ na variável $A[i]$. Resta utilizar a característica do corpo nos polinômios para verificar se é, ou não, uma identidade de $M_{k, l}(E)$. Testamos inicialmente para $\operatorname{char}(K)=p=3$ usando a função mod do Maple, a qual avalia uma expressão sobre os inteiros módulo $p$. Por exemplo, $5 \bmod 3=2$ e $(-7 x+4 y) \bmod 5=3 x+4 y$. Dessa forma, basta fazer $A[i] \bmod 3$ para cada $i=2, \ldots, 9$. Com isso, tivemos como resultado que o menor grau $m$ tal que $S t_{m}$ seja uma identidade polinomial para $M_{1,1}(E)$ é $m=6$.

De fato, analisando todos os coeficientes de cada entrada de $S t_{6}$, (podemos usar a função coeffs) obtemos

coeficientesAl:=\{\};

for i to 2 do;

for $j$ to 2 do;

coeficientesA1: $=\{$ op $($ coeficientesA1 $), \operatorname{coeffs}(A[1][i, j]) ;\}$

end do;

end do:

coeficientesA1; 


$$
\{-36,36\}
$$

ifactor(coeficientesAl);

$$
\left\{(2)^{2}(3)^{2},-(2)^{2}(3)^{2}\right\}
$$

Assim, como $36=2^{2} \cdot 3^{2}$ (utilizamos a função ifactor $(x)$ para fatorar facilmente um número inteiro $x$ ), não há primos que o divida além do 2 e 3 . Note que quando $p=2$, St $t_{6}$ também é uma identidade de $M_{k, l}(E)$, o que é óbvio, pois nesse caso $E$ torna-se comutativa e, pelo teorema de Amtsur e Levitzki, $S t_{4}$ é uma identidade polinomial de $M_{k, l}(E)$.

Fazendo essa análise para os polinômios de grau $n=2, \ldots, 9$ obtemos os resultados da tabela abaixo:

\begin{tabular}{|c|c|c|}
\hline n & Coeficientes & Fatoração \\
\hline 2 & $\{-1,1\}$ & $\{-1,1\}$ \\
\hline 3 & $\{-2,-1,1,2\}$ & $\{-1,1,-(2),(2)\}$ \\
\hline 4 & $\{-4,4\}$ & $\left\{(2)^{2},-(2)^{2}\right\}$ \\
\hline 5 & $\{-12,-8,-4,4,8,12\}$ & $\left\{(2)^{2},(2)^{3},(2)^{2}(3),-(2)^{2},-(2)^{3},-(2)^{2}(3)\right\}$ \\
\hline 6 & $\{-36,36\}$ & $\left\{(2)^{2}(3)^{2},-(2)^{2}(3)^{2}\right\}$ \\
\hline 7 & $\{-144,-108,-36,36,108,144\}$ & $\left\{\begin{array}{l}(2)^{2}(3)^{2},(2)^{2}(3)^{3},(2)^{4}(3)^{2} \\
-(2)^{2}(3)^{2},-(2)^{2}(3)^{3},-(2)^{4}(3)^{2}\end{array}\right.$ \\
\hline 8 & $\{-576,576\}$ & $\left\{(2)^{6}(3)^{2},-(2)^{6}(3)^{2}\right\}$ \\
\hline 9 & $\{-2880,-2304,-576,2304,2280\}$ & $\left\{\begin{array}{l}(2)^{6}(3)^{2},(2)^{8}\left(3^{2}\right),(2)^{6}(3)^{2}(5) \\
-(2)^{6}(3)^{2},-(2)^{8}\left(3^{2}\right),-(2)^{6}(3)^{2}(5)\end{array}\right.$ \\
\hline
\end{tabular}

Tabela 3: Coeficientes das entradas do polinômio standard de grau $n$

Pela conjectura de [18], para $n=2$, devemos ter $d_{2}=(2 \cdot 2-1) p-1=3 p+1$. Ou seja, se $p=3$ então $d_{2}=10$ é o grau mínimo da identidade standard para $M_{n}(E)$. Nos parece um valor alto já que $S t_{6} \in T(R)$. Sabemos que, se $S t_{n}$ é uma identidade polinomial para uma álgebra $A$, então $S t_{n+1} \in T(A)$. Isso justifica o fato de $S t_{i}$, com $i=7,8,9$ ser uma identidade para $R$, uma vez que $S t_{6}$ o é.

Note que $6=2 \cdot 2+3-1$. Logo, a resposta da primeira questão dada em [1], para a álgebra $R$, é afirmativa, isto é, existe $m \in\left[2 n+p-1, p n^{2}+1\right]$ tal que $\operatorname{Stm} \in T(R)$. Além disso, mostra-se $d_{2}=3 \cdot 3+1=10$ não é uma identidade minimal, ou seja, a conjectura é falsa para $n=2$ e $p=3$ (para a álgebra $R$ ). Porém, se $p=5$, temos que $8=2 \cdot 2+5-1$ e $S t_{8}$ não é uma identidade para $R$. Consequentemente, isso implica que $S t_{8}$ também não é uma identidade polinomial de $M_{2}(E)$. 
Conclui-se que o limite inferior do intervalo $\left[2 n+p-1, p n^{2}+1\right]$ para $M_{n}(E)$ não é o melhor. De fato, pela Tabela 3 temos que nenhum dos polinômios calculados pode ser uma identidade de $R$ $\operatorname{para} \operatorname{char}(K)=p>3$.

\section{OUTROS RESULTADOS SOBRE O POLINÔMIO STANTARD}

Da forma como construímos os procedimentos, é muito simples tornar qualquer variável anticomutativa. Dessa forma, definimos $x, y, z, w$ como variáveis anticomutativas e consideramos os seguintes conjuntos

$R_{1}=\left\{\left[\begin{array}{ll}a & b \\ c & d\end{array}\right] ; a, b, c, d \in E_{0}\right\} ; R_{2}=\left\{\left[\begin{array}{ll}a & y \\ z & d\end{array}\right] ; a, d \in E_{0}, y, z \in E_{1}\right\} ;$
$R_{3}=\left\{\left[\begin{array}{ll}x & b \\ c & w\end{array}\right] ; x, w \in E_{1}, b, c \in E_{0}\right\} ; R_{4}=\left\{\left[\begin{array}{cc}x & y \\ z & w\end{array}\right] ; x, y, z, w \in E_{1}\right\}$.

Assim, como $E=E_{0} \oplus E_{1}$, temos que a álgebra $M_{2}(E)=R_{2} \oplus R_{3}=R_{1} \oplus R_{4}$. Os resultados obtidos até a seção anterior foram em relação às álgebras $R_{1}$ e $R_{2}$. Para a álgebra $R_{1}$, vale o teorema de Amitsur e Levitzki (ver a Equação 2.5). Nesse sentido, calculamos os polinômios standard de grau $3<m<9$ apenas para $R_{4}$ e $R_{2}+R_{3}=\left\{u+v ; u \in R_{2} e v \in R_{3}\right\}$ e obtemos que $S t_{6} \in T\left(R_{4}\right)$ quando $\operatorname{char}(K)=3$. Além disso, não obtemos nenhuma outra identidade polinomial para $R_{i}, i=1,2,3,4$.

Entretanto, podemos obter algumas identidades polinomiais essencialmente fracas para $M_{2}(E)$, isto é, identidades polinomiais fracas que não são identidades para $M_{2}(E)$. Vale lembrar que a definição de identidade fraca para matrizes com entradas em $K$ pode ser naturalmente estendida para as matrizes com entradas em $E$ (ver [25]).

Dessa forma, sejam $u_{1}, u_{2}, u_{3} \in R_{1}$. Verificamos que

$$
S t_{4}\left(u_{1}, u_{2}, u_{3}, u_{4}\right)=0 \text { e } S t_{6}\left(u_{1}, u_{2}, u_{3}, u_{4}, u_{5}, u_{6}\right)=0
$$

onde $u_{4}, u_{5}, u_{6} \in R_{4}$.

Uma vez que $M_{2}(E)=R_{1} \oplus R_{4}$, podemos dizer que $S t_{4}$ e $S t_{6}$ são identidades polinomiais essencialmente fracas para $M_{2}(E)$. Podemos dizer ainda que estas são identidades polinomiais 2graduadas de $M_{2}(E)$ (termo não definido no presente texto, para mais detalhes ver [4]). Porém, o mesmo não acontece para $S t_{5}\left(u_{1}, u_{2}, u_{3}, u_{4}, u_{5}\right)$ e $S t_{7}\left(u_{1}, u_{2}, u_{3}, u_{4}, u_{5}, u_{6}, u_{7}\right) \operatorname{com} u_{4}, u_{5}, u_{6}, u_{7} \in$ $R_{4}$, isto é, quando o grau do polinômio standard é ímpar.

Se $u_{i} \in R_{1}$, com $i=1, \ldots, 4$, temos que todos os polinômios de grau maior que 4 se anulam independentemente da característica do corpo, o que é esperado devido à linearidade do polinômio standard e o teorema de Amitsur e Levitzki. 


\section{CONCLUSÃO}

Após a leitura de vários trabalhos sobre as álgebras com identidades polinomiais, começamos a construção de modelos computacionais para trabalhar com álgebras não comutativas. Dentre esses modelos, construímos procedimentos, com uso do programa Maple, para satisfazer a propriedade distributiva da multiplicação sobre a soma, inerente ao estudo de álgebras em geral, bem como para satisfazer propriedades de álgebras específicas tais como a álgebra de Grassmann.

Como nossos principais objetos de pesquisa foram as álgebras matriciais $M_{n}(E)$ e $M_{k, l}(E)$, as quais, em geral, temos dificuldades em encontrar suas identidades, buscamos encontrar e verificar as identidades polinomiais de tais álgebras em seus casos particulares como, por exemplo, $n=2$ e $k=l=1$. De posse dos procedimentos construídos, verificamos a validade das identidades polinomiais da álgebra de Gassmann dada por $\left[\left[x_{1}, x_{2}\right], x_{3}\right]$, a identidade de Hall para a álgebra $M_{2}(K)$ dada por $\left[\left[x_{1}, x_{2}\right]^{2}, x_{2}\right]$ e o bem conhecido Teorema de Amtsur e Levitzki, o qual afirma que o polinômio standard de grau $2 n$ dado por $s t_{2 n}\left(x_{1}, \ldots, x_{2 n}\right)=\sum_{\sigma \in S_{2 n}}(-1)^{\sigma} x_{\sigma(1)} \ldots x_{\sigma(2 n)}$ é uma identidade para a álgebra $M_{n}(K)$.

Devido às boas propriedades do polinômio standard, implementamos um procedimento no Maple para encontrar identidades standard para a álgebra $M_{k, l}(E)$, em especial quando $k=l=1$. Identificamos que o polinômio standard de grau 6 é uma identidade de $M_{1,1}(E)$, quando a característica do corpo é $p=3$. Quando o $p>3$ não encontramos identidades polinomiais de grau menor que 10, visto que não conseguimos calcular polinômios standard de grau maior que 9 devido ao tempo de processamento ser muito grande. Com isso, apesar de construirmos procedimentos genéricos, não conseguimos encontrar identidades para álgebra das matrizes $M_{k, l}(E)$, com $k, l>1$ e $p>3$.

Porém, pudemos encontrar identidades polinomiais essencialmente fracas de $M_{n}(E)$, isto é, polinômios que não são identidades de $M_{n}(E)$, mas são identidades para algum subespaço da mesma. Verificamos, por exemplo, que $s t_{4}\left(x_{1}, x_{2}, x_{3}, x_{4}\right)=0$ e $s t_{6}\left(x_{1}, x_{2}, x_{3}, x_{4}, x_{5}, x_{6}\right)=0$ com $x_{1}, x_{2}, x_{3} \in M_{2}\left(E_{0}\right)$ e $x_{4}, x_{5}, x_{6} \in M_{2}\left(E_{1}\right)$, onde $E_{0}, E_{1}$ são subespaços da álgebra de Grassmann E.

Além disso, percebemos que calcular o polinômio standard de grau 10 para $M_{n}(E)$ seria interessante a ponto de verificar a validade ou não da conjectura de Kemer quando $p=3$. Porém, ficou inviável computacionalmente, devido ao tempo de processamento ser alto. Foram realizadas algumas tentativas de paralelização do procedimento para obter o polinômio standard. Porém, os resultados não foram satisfatórios, sendo que o tempo de processamento, por razões relativas ao acesso de memória, foi maior que o esperado.

Estamos revisando esse procedimento, tendo em vista que a programação paralela é uma das saídas que temos para o cálculo do polinômio standard de grau $n>9$.

\section{ACKNOWLEDGEMENTS}

Agradecimentos à Fundação de Amparo à Pesquisa do Estado da Bahia (FAPESB). 


\begin{abstract}
In this paper is presented a computational approach to deal with algebras that satisfy polynomial identities. More precisely, we used the Maple program to verify and identify polynomial identities of matrix algebras with entries in the Grassmann algebra $E$, especially the algebra $M_{k, l}(E)$, which Di Vincenzo and La Scala showed interesting results when $k=l=1$, using the notion of weak polynomial identities. Some procedures were created in Maple to suit the product of the matrices according to the properties of $E$, this being a non-commutative algebra. In addition, we implemented some functions with shorter processing time in solving certain problems compared to similar Maple functions. It should be noted that we use the Maple version 18 on a computer with Intel $\AA$ Core $i 7$ and 8GB of RAM memory. We conclude with a study of the conjecture given by Kemer at about the minimum degree of the standard polynomial for the algebra $M_{n}(E)$.
\end{abstract}

Keywords: PI-algebras; Maple; Algebraic computing; Weak Polynomial Identities.

\title{
REFERÊNCIAS
}

[1] S.M. Alves, K.K. Sartori \& V.A. Arakawa. The Standard Identity on $M_{n}(E)$ in Characteristic $p>2$. International Journal of Algebra, 9(4) (2015), 155-158.

[2] S.A. Amitsur \& J. Levitzki. Minimal Identities for Algebras. Math. Soc. 1, (1950), 449-463.

[3] L.N. Andrade. "Introdução à Computação Algébrica com o Maple”. SBM (2004).

[4] S.S. Azevedo. "Identidades Graduadas para Álgebras de Matrizes”. Tese de doutorado, Instituto de Matemática, Estatística e Computação Científica (UNICAMP) (2003).

[5] M. Dehn. Über die Grundlagen der Projektiven Geometrie und Allgemeine Zahlsysteme. Math. Ann.85, (1922), 184-194.

[6] V. Drensky. New Central Polynomials for the Matrix Algebra. J. Math., 92 (1995), 235-248.

[7] V. Drensky \& G.P. Cattaneo. A Central Polynomial of low Degree for $4 \times 4$ Matrices. J. Algebra, 168 (1995), 469-478.

[8] V. Drensky \& T.G. Rashkova. Wear Polynomial Identities for the Matrix Algebras. Commun. in Algebra, 21 (1993), 3779-3795.

[9] E. Formanek. Central Polynomials for Matrix Rings. J. Algebra, 23 (1972), 129-132.

[10] E. Formanek. The Polynomial Identities and Invariants of $n \times n$ Matrices. CBMS Regional Conf. Series in Math., 78 (1991).

[11] A.T. Galão. "PI-Álgebras". Dissertação de mestrado, Universidade Estadual de Campinas, Campinas, SP (2003).

[12] A. Giambruno \& A. Valenti. Central Polynomials and Matrix Invariants. Israel J. Math., 96 (1996), 281-297.

[13] P. Halpin. Central and Wear Identities for Matrices. Commun. in Algebra, 11 (1983), 2237 - 2248. 
[14] N. Jacobson. Structure theory of algebraic algebras of bounded degree. Annals of Math., 441(46) (1945), 1-3.

[15] N. Jacobson. "PI-Algebras: An Introduction", volume 141. Lectures Notes in Math., Springer-Verlag, Berlin - New York (1975).

[16] I. Kaplansky. Problems in the Teory of Rings. Report of a conference on linear algebras, 502 (1957), $1-3$.

[17] I. Kaplansky. Problems in the Theory of Rings, Revisited. The American Mathematical Monthly, 77(5) (1970), 445-454.

[18] A. Kemer. Matrix Type of Some Algebras over a Field of Characteristic p. Journal of Algebra, 251(2) (2002), 849-863. doi:10.1006/jabr.2001.9113.

[19] P. Koshlukov \& T.C. Mello. On the Polynomial Identities of the Algebra $M_{11}(E)$. Linear Algebra and its Applications, 438(2010) (2013), 4469-4482.

[20] A. Popov. Identities of the Tensor Square of a Grassmann Algebra. Algebra i Logika, 4 (1982), 442 471.

[21] R. Portugal. Introdução ao Maple (2002). Laboratório Nacional de Computação Científica (LNCC).

[22] Y.P. Razmyslov. Finite Basing of the Identities of Matrix Algebra Second Order Over a Field of Characteristc Zero. Translation: Algebra and Logica 12, (1973), 47-73.

[23] Y.P. Razmyslov. On a Problem of Kaplansky. Translation: Math. USSR, IZv., (1973), 479-496.

[24] L.H. Rowen. Polynomial Identities of Rings Theory. Acad. Press., (1980).

[25] O.M. Vincenzo \& R. Scala. Robinson-Schensted-Knuth Correspondence and Wear Polynomial Identities of $M_{1 ; 1}(E)$. Algebra Colloquium, 12(2) (2005), 333-349.

[26] W. Wagner. Über die Grundlagen der Projektiven Geometrie und Allgemeine Zahlsysteme. Math. Ann.113, (1936), 528-567. 